
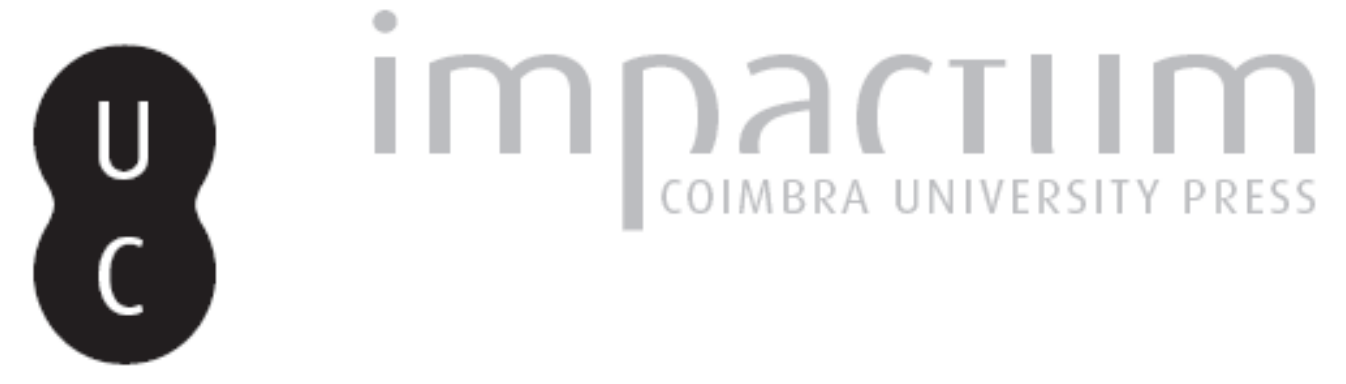

\title{
¿Lucius Cornelius Bocchus, un lusitano miembro del ordo equester y escritor? Una feliz intuición de Emil Hübner reforzada y difundida por Theodor
}

Autor(es): $\quad$ Mayer I. Olivé, Marc

Publicado por: Faculdade de Letras da Universidade de Coimbra/Imprensa da

URL

persistente:

DOI: $\quad$ DOI:https://doi.org/10.14195/1647-8657_55_13

Accessed : $\quad$ 26-Apr-2023 09:42:57

A navegação consulta e descarregamento dos títulos inseridos nas Bibliotecas Digitais UC Digitalis, UC Pombalina e UC Impactum, pressupõem a aceitação plena e sem reservas dos Termos e Condições de Uso destas Bibliotecas Digitais, disponíveis em https://digitalis.uc.pt/pt-pt/termos.

Conforme exposto nos referidos Termos e Condições de Uso, o descarregamento de títulos de acesso restrito requer uma licença válida de autorização devendo o utilizador aceder ao(s) documento(s) a partir de um endereço de IP da instituição detentora da supramencionada licença.

Ao utilizador é apenas permitido o descarregamento para uso pessoal, pelo que o emprego do(s) título(s) descarregado(s) para outro fim, designadamente comercial, carece de autorização do respetivo autor ou editor da obra.

Na medida em que todas as obras da UC Digitalis se encontram protegidas pelo Código do Direito de Autor e Direitos Conexos e demais legislação aplicável, toda a cópia, parcial ou total, deste documento, nos casos em que é legalmente admitida, deverá conter ou fazer-se acompanhar por este aviso.

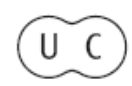


CONIMBRIGA

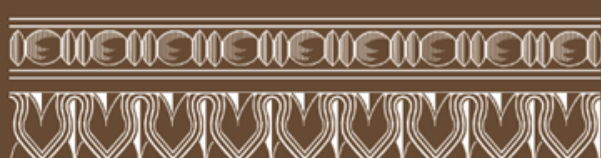

INSTITUTO DE ARQUEOLOGIA

VOLUME LV • 2016

FACULDADE DE LETRAS
UNIVERSIDADE DE COIMBRA 
MARC MAYer I Olivé

Institut d'Estudis Catalans / Universitat de Barcelona

mayerolive@yahoo.es

¿LUCIUS CORNELIUS BOCCHUS, UN LUSITANO MIEMBRO DEL ORDO EQUESTER Y ESCRITOR? UNA FELIZ INTUICIÓN DE EMIL HÜBNER REFORZADA Y DIFUNDIDA POR THEODOR MOMMSEN DE NUEVO A EXAMEN.

WAS LUCIUS CORNELIUS BOCCHUS A LUSITANIAN MEMBER OF THE ORDO EQUESTER AND WRITTER? A NEW APPROACH TO AN EMIL HÜBNER'S BRILLIANT INTUITION, REINFORCED AND SPREAD BY THEODOR MOMMSEN.

“Conimbriga” LV (2016) p. 251-267

https://doi.org/10.14195/1647-8657_55_13

ABstract: Hübner's proposal to identify the Bocchi, present as a literary source in Pliny and Solinus, with one of the Cornelii Bocchi documented by the Lusitanian inscriptions has had great fortune and acceptation. The aim of this paper is to notice up to what point this fact is a conjectural supposition and a question that must remain opened despite the considerable progress adrieved.

Keywords: Roman History, Epigraphy, Lusitania, Cornelii Bocchi, Pliny the Elder, Solinus.

RESUMEN: La propuesta de Hübner de identificar a los Bocchi, presentes como fuente en Plinio y en Solino, con uno de los Cornelii Bocchi documentados por las inscripciones de Lusitania, ha tenido una gran fortuna y aceptación. La intención de este trabajo es notar hasta qué punto se trata de una cuestión de carácter conjetural que debe quedar abierta, a pesar de los grandes progresos realizados.

Conimbriga, 55 (2016) 251-267 
Palabras Clave: Historia romana, Epigrafía, Lusitania, Cornelii Bocchi, Plinio el Viejo, Solino. 


\section{¿LUCIUS CORNELIUS BOCCHUS, UN LUSITANO MIEMBRO DEL ORDO EOUESTER Y ESCRITOR? UNA FELIZ INTUICIÓN DE EMIL HÜBNER REFORZADA Y DIFUNDIDA POR THEODOR MOMMSEN DE NUEVO A EXAMEN*.}

Será necesario detenerse antes de entrar, aunque sea brevemente, en analizar el expediente epigráfico de Lucius Cornelius Bocchus ${ }^{1}$, documentado en diversas ciudades de Lusitania y que tanto ha llamado la atención de los estudiosos en tiempo reciente ${ }^{2}$. Convendrá para ello fijarnos primeramente en lo que ha sido el eje de los últimos estudios, que se centra en la identificación de este personaje con el Bocchus o, quizás mejor, los Bocchi citados por Plinio el Viejo y Solino.

Cabe destacar al respecto en este caso además que fue precisamente la autoridad del gran epigrafista e historiador Theodor Mommsen, quien aceptaba una propuesta de su colega el erudito latinista y epigrafista Emil Hübner, la que dió pie a esta identificación. Un hecho que en el momento actual parece ser considerado indiscutible por parte de los estudiosos, aunque evidentemente en términos filológicos no pueda ser considerado más que como una feliz conjetura que presenta, sin duda, muchos visos de realidad y que proporcionaría, de ser cierta, un notable

${ }^{*}$ El presente trabajo se ha realizado en el marco del proyecto FFI2015-68571-P, en el seno del Grup consolidat LITTERA 2014SGR63 de la UB y del programa Corpus Inscriptionum Latinarum del IEC.

${ }^{1}$ Cf. IRCP ve una mención a los Bocchi en los núms. 185, 188, 189, 205, 207 y 351. El reciente Atlas 2003: 115-116, s.v. Bocchus, señala ya siete inscripciones referidas a los mismos, añadiendo Ficheiro Epigrafico 275: L. Cornelio Boccho L. f. Gal. Salaciensi, hallada en Lisboa. Ahora ENCARNAÇÃo 2011: 189- 201 y AlARCÃo 2011: 329-336.

${ }^{2}$ Cardoso y Almagro 2011. Resulta fundamental por su completa documentación Almagro 2011:25-56, con una bibliografía anterior punto menos que exhaustiva.

Conimbriga, 55 (2016) 251-267 
elemento al no muy extenso elenco de escritores documentados directamente por la epigrafía.

El entrecruzamiento entre los Bocchi epigráficos, documentados en la Lusitania, y los citados por los autores latinos, Solino y Plinio el Viejo, si fueran en realidad más de uno, se volvió casi insoslayable cuando Th. Mommsen se inclinó a creer que un Cornelius Bocchus lusitano era el escritor citado por Solino ${ }^{3}$, siguiendo la sugerencia de E. HÜBNER ${ }^{4}$.

${ }^{3}$ Th. Mommsen, C. Iulii Solini collectanea rerum memorabilium, Berlin 1864, dice en su prefacio a la edición, p. XVII: "Bocchus autem hic Solinianus haud scio an sit Cornelius Bocchus is, quem inter auctores Latinos recenset Plinius in indicibus 1. 16. 33. 34. 37 quemque citat cum propter gemmas quasdam Hispanicas omnes $(37,24$. 97. 127) tum ad originem Sagunti declarandam conditi secundum Bocchum a Zacynthiis anno CC ante excidium Troiae $(16,216)$. Haec adnotatio licet mire conveniat cum Solinianis, tamen Bocchi apud Plinium relata cum omnia pertineant ad Hispania neque omnia locum habere potuerint in opere chronographico, magis putarim Plinium eiusdem viri libro diverso fortasse de admirandis Hispaniae usus esse". La segunda edición a cargo del mismo Mommsen aparece al año siguiente con un cambio de editor, C. Iulii Solini collectanea rerum memorabilium, iterum recensuit Th. Mommsen, Berlin 1895, intercala a continuación del excidium Troiae, p. XIV: "quemque probabile est ipsum esse cui dedicati sunt tituli duo in Lusitania inventi (cf. Huebner in Hermae vol. 1 p. 397) C.I.L. II, 35: L. Cornelio C. f. Boccho flam(ini) provin(ciae), tr(ibuno) mil(itum) colonia Scalabitana ob merita in colonia [m] et II, 5184: [L. C]ornelio L. (vel C.) f. [B] occho [flamin] i provinc(iae) [tr(ibuno)]mil(itum) leg(ionis) III Aug(ustae). Bocchiana apud Plinium relata licet in quibusdam mire conveniant cum Solinianis, tamen cum omnia pertineant ad Hispaniam neque omnia locum habere potuerint in opere chronographico, magis putarim Plinium non eo usum esse, sed eiusdem viri libro diverso fortasse de admirandis Hispaniae", p. 25, 9. 34, 11, 36, 2. EE I, p. 182: Referendus est titulus summa cum probabilitate ad Cornelium Bocchum historicum, cuius chronica Solinus excerpsit, postquam eius de admirandis fortasse Hispaniae libro librisve iam Plinius usus est frequenter. PLIN. Nat. 37, 24; 3797 y 37, 127 y en los índices de los libos $16,33,34,37$.

${ }^{4}$ HÜBNER 1866: 397, donde dedica media página al comentario de la noticia sobre Bocchus escritor de la p. XVII de la $1^{\mathrm{a}}$ ed. de Solino de Mommsen, donde se muestra convencido de la identidad del Bocchus citado por Solino y el presente en Plinio el Viejo para acabar concluyendo después de mencionar los tituli de Salacia: "An der Identität dieses Bocchus mit den Schrifteller wird nicht zu zweifeln sein, den die Zeit der Inschrift (sie gehört ihrer ganzen Fassung nach und weil beim Tribunentitel die Angabe der Legion fehlt in die Augusteische Zeit) und der Fundort (vielleicht war Bocchus von Geburt ein Lusitaner; der Name ist in jenen Gegenden häufig) stimmen durchaus". Una afirmación taxativa que conviene revisar quizás frente a los datos de la epigrafía.

Conimbriga, 55 (2016) 251-267 
Los artículos correspondientes de la $R E$ han seguido, implícita o explícitamente, la posición de MOMMSEN y de HÜBNER ${ }^{5}$.

Pero, si queremos remontarnos al origen mismo de la cuestión, recordemos que TH. Mommsen precisa y repite en ambas y sucesivas ediciones que: "Bocchus autem hic Solinianus haud scio an sit Cornelius Bocchus is, quem inter auctores Latinos recenset Plinius in indicibus 1. 16. 33. 34. 37 quemque citat cum propter gemmas quasdam Hispanicas omnes $(37,24.97 .127)$ tum ad originem Sagunti declarandam conditi secundum Bocchum a Zacynthiis anno CC ante excidium Troiae (16, 216)". Vemos por consiguiente como Mommsen pone de manifiesto sus reservas sobre la probable identidad entre los dos Bocchi, el de Solino y el de Plinio, ya que no está seguro de que ambos autores hayan tenido en la mano la misma obra de Bocchus, lo que le permite suponer que quizás Plinio tuvo en su manos unos "admiranda Hispaniae", que serían distintos del tratado geográfico que manejaría Solino y que ambas obras podrían incluso depender de dos distintos Bocchi. Resulta claro, sin embargo, que cree que es altamente probable que se trate del personaje mencionado en las dos inscripciones lusitanas que aquí comentaremos.

Si nos atenemos ahora al expediente epigráfico, veremos que tampoco está exento de problemas ya que los personajes que llevarían el nombre de Lucius Cornelius Bocchus serían dos: uno de ellos Luci filius y el otro Caii filius, aunque los epigrafistas a partir de Emil Hübner han tendido, siguiendo la opinión de éste, a reducirlos a un mismo personaje suponiendo un posible error de transmisión.

Veamos el origen y la evolución de esta espinosa cuestión. E. HÜBNER, en sus Additamenta al CIL $\mathrm{II}^{6}$ refiriéndose a una inscripción de Caetobriga, hallada en Troya en 1871, "in situ parietinarum", cerca de Setúbal, nota que complementaba la noticia epigráfica de Alcácer do Sal, Salacia, en la que un personaje homónimo, pero con distinta filiación, es honrado por la colonia Scallabitana, Santarém7 . A partir de

${ }^{5}$ RE 5, s.v. "Bochus 3" (Henze), Stuttgart 1897col. 579, acepta la propuesta de Mommsen sobre el contenido hispánico de su obra recogida por Solino que corresponde a un resumen de otra obra que habría visto también Plinio, y la identificación de Hübner; cf. además RE 7, s. v. "Cornelius 77 L. Cornelius Bocchus" (A. Stein), Stuttgart 1900, col. 1273, que remite al artículo anterior limitándose a consideraciones prosopográficas.

${ }^{6}$ HÜBNER 1872:182-183, núm. 291.

${ }^{7}$ CIL II 35 .

Conimbriga, 55 (2016) 251-267 
estos datos E. Hübner sospechó que se trataba del mismo personaje y que pudo haber habido un error de copia por parte de Cornide y Clusius, de quienes depende la lectura de este último epígrafe. En la edición de estos mismos fragmentos en CIL II 5184, aunque repite el texto de los additamenta, incluyó una nueva frase en la que manifestaba que se trataba efectivamente del mismo personaje y que, en consecuencia, había que tener más en cuenta la posibilidad de error por parte de sus fuentes: "Itaque Caetobriga, quod erat oppidum vetustum ad mare situm ab Olisipone meridiem versus, aut oriundus esse aut praedia ibi habuisse putandus erit Bocchus rerum scriptor ibique etiam statuis honoratus esse a variis oppidis Lusitanis"

Resulta muy significativo el comentario de Robert Étienne a las inscripciones de los Bocchi al tratar de los flamines provinciales ${ }^{10}$. Se desprende de dicho comentario que considera la existencia de dos flamines distintos: L. Cornelius C. f. Bocchus (CIL II $35=I L S$ 2920) y L. Cornelius L. f. Bocchus (CIL II $5184=$ ILS 2921), siguiendo para la filiación de este último la lectura sobre fotografia de J. LeITE DE VASCONCELLOS $^{11}$ y descartando así la corrección onomástica como $C$. $f$. propuesta por H. DesSAU en $I L S^{12}$ y HeNZE en $R E$ III, 1897, col. 579, y seguida por $P I R^{2}$ C 1333, p. 312. R. ÉTIENNE se extiende sobre quién podría ser de estos dos personajes la fuente de Plinio el Viejo, condición que generalmente se atribuía al primero (CIL II 35), mientras que él se inclina a creer, de acuerdo con lo expuesto en M. Schanz, y C. Hosius $^{13}$, que quizás éste no fuera, como quería Mommsen, la fuente de

${ }^{8}$ CIL II p. 803, "Puto hominem indicari in utroque titulo eundem, cum dignitates prorsus conveniant".

${ }^{9}$ HÜBNER 1872: 183.

${ }^{10}$ ÉtIENNE 1974: 122-124.

${ }^{11}$ Vasconcellos 1895: 71.

${ }^{12}$ H. Dessau en $I L S$ I, p. 569 propone al comentar CIL II $35=I L S 2920$ y CIL II 5184 = ILS 2921: "Videtur esse Cornelius Bocchus, ex cuius libro aliquo Plinius complura hausit de Hispania (h. n. 16, 246) et potissimum de Lusitania (h. n. 37, 24. 97. 127). Bocchus citatus a Solino (cf. Mommsen praef. Ad Solinum p. XVII) num idem sit incertum", lo cual viene a demostrarnos de nuevo las vacilaciones que desde un primer momento despertó la conjetura de Mommsen.

${ }^{13}$ Schanz, Hosius, Krueger 1935: 646-647 § 440, 4, Duda que los Bocchi de Plinio y Solino sean el mismo autor y en el caso del Cornelius Bocchus fuente de Plinio acoge la hipótesis de la identificación con el personaje de Salacia propuesta anteriormente. Precedentemente Schanz 1901: $256 \S 440,4$, aceptaba lo propuesto por 
Solino, ya que la obra de este autor vió la luz en el 49 d.C. bajo Claudio; hecho que según R. ÉTIENNE permitiría suponer al segundo (CIL II 5184 = ILS 2921) como fuente únicamente de Plinio, en época de Nerón o Vespasiano; deja en cambio la posibilidad de que L. Cornelius $L$. $f$. Bocchus (CIL II $5184=$ ILS 2921) pudiera ser además hijo del primero en una clara imprecisión. Plantea además ÉTIENNE la dificultad de identificar a uno de los dos personajes con el mencionado en CIL II 5617, que dependería para aproximarlo a uno u a otro de la identificación de los Caesares cuya praefectura se menciona en la inscripción: Augusto y Tiberio o Vespasiano y Tito.

Desde un punto de vista prosopográfico H. DeviJver mantuvo con prudencia la distinción de ambos personajes mencionados en las inscripciones ${ }^{14}$ y no insiste en la propuesta hecha por R. ETIENNE de considerar un parentesco entre ambos personajes como padre e hijo ${ }^{15}$. DeviJver anota además: "Valde incertum an hic Bocchus (C 228) an alter Bocchus (C 229) idem sit ac "auctor Bocchus" quem Plinius (n.h. $16,216 ; 37,24,97,127)$ et Solinus $(1,92 ; 2,11 ; 2,18)$ memorant" ${ }^{\prime 16}$, una prudente toma de distancia respecto a un problema de difícil solución. En la misma línea de H. DeviJver se había mantenido, creemos que con razón, Y. LE BoHEC en su trabajo ${ }^{17}$.

La aportación más substancial a esta cuestión ha sido la de $\mathrm{J}$. D'ENCARNAÇão, que con una documentación exhaustiva, replanteó la situación añadiendo una nueva inscripción a la serie y aproximan-

Th. Mommsen y H. Peter. Véase además Peter 1906: CXXIII-CXXV y 94-95 para los fragmentos, donde de nuevo pone de relieve, p. CCXXIII, la autoridad de Mommsen y la contribución de E. Hübner al respecto.

${ }^{14}$ Cf. DviJver 1976: 292, C. 228 , para CIL II 2479= 5617; y 292-293 C. 229, para $A E$ 1967, 195 ad CIL II $5184=$ ILS 2921 y CIL II $35=I L S 2920$, donde se pregunta si el personaje de la primera inscripción no será el hijo del de la segunda.

${ }^{15}$ ÉTIENNE 1974:123-124.

${ }^{16}$ DeviJver 1993: 2080.

${ }^{17}$ Le Bohec 1989: 136 , donde lo sitúa en torno a la época de Claudio e indica: “Ce Bocchus a parfois été identifié avec le géographe utilisé par Pline L'Ancien (H.N. XVI, XXXIII.XXXIV et XXXVIII) et par Solin”. Además DeviJver 1993: 2078-2080, data L. Cornelius C. f. Bocchus (IRCP núm. 185=AE 1967, $195=$ CIL II $35=$ ILS 2920) en la época de Tiberio o Claudio (cf. sobre el flaminato de este Bocchus Fishwick 2002:152, núm. 4) y a L. Cornelius L. f. Bocchus al final del reinado de Nerón o a inicios del de Vespasiano.

Conimbriga, 55 (2016) 251-267 
do otra muy probable ${ }^{18}$. Recordemos que anteriormente el mismo E. HÜBNER había corregido la falsa localización y lectura de CIL II 2479 $(=5617)$ devolviéndola de Valdereis a Alcácer do $\mathrm{Sal}^{19} \mathrm{y}$ restituyendo en la misma el nombre L. Cornelius L. f. Boc]chus, siguiendo en ello a J. LEITE DE VASCONCELlOS ${ }^{20}$; la misma ubicación acepta y comenta acertadamente J. D'ENCARNAÇÃO, que prefiere restituir, no obstante, la filiación $C$ (ai) $f(\text { ilius })^{21}$. Este autor, que se ocupa en su corpus asimismo de CIL II 35 y de CIL II 518422, llega a conclusiones que no pueden ser más prudentes: por una parte duda sobre el grado de parentesco que media entre los Bocchi documentados por la epigrafía lusitana y por otro considera una cuestión abierta la hipótesis de que se pueda tratar, en el caso de L. Cornelius C. f. Bocchus, del historiador mencionado por Plinio como sugirió E. HüBNER. Sin embargo, la restitución o, si se quiere, corrección que hace a $C I L$ II 5184, entendiendo C(ai) f(ilio) en lugar de $L$ (uci) $f(i l i o$ ) implicaría en principio la posibilidad de que puedan identificarse la mayor parte de los documentos como referidos a un mismo personaje, hecho que del texto del autor no se desprende, ya que comenta que la homonimia no puede ser considerada como un criterio irrefutable y que la hipótesis de E. HüBNER no deja de ser seductora, aunque sin ir más allá en su comentario.

Hay que señalar también que ya con anterioridad F. BANDEIRA DE Almeida, había documentado CIL II 5184 en la Quinta do Solar da Sempre Noiva cerca de Evora, y había propuesto la identidad con la de Alcácer do Sal, discutiendo en cambio que pudiera atribuirse al historiador Cornelius Bocchus ${ }^{23}$. E. Hübner parece haber preferido suponer la forma $L$ (uci) $f($ ilio), a pesar de las dudas de Augusto Soromenho, que le mandó la copia del texto, respecto a la filiación, proponiendo en cambio, que el error pudiera radicar quizás en una mala copia por parte

${ }^{18}$ IRCP 188, p. 262 (= AE 1985, 499, cf. LÁZARo FARIA 1984: 14-15) y núm. 205, p. 205, respectivamente.

${ }^{19}$ HÜBNER 1899: 356, núm. 4.

${ }^{20}$ VASCONCELLOS 1895: 69-76 y VASCONCELLOS 1914: 306-307.

${ }^{21}$ IRCP 189, pp. 262-264 (= CIL II 2479 y 5617)

${ }^{22}$ IRCP 185 (= CIL II 35, ILS 2920), pp. 257-258; y 207 (= CIL II 5184, AE 1967, 195 e ILS 2921), pp. 276-277.

${ }^{23}$ BANDEIRA FERREIRA 1956: 87-105; cf. IRCP, 277, núm.185, para esta cuestión y la atribución errónea de la pieza por EsPanca 1966: 369 al templo de Santana de Campos en Arraiolos.

Conimbriga, 55 (2016) 251-267 
de Cornide de CIL II 35, donde leyó C(ai) filio ${ }^{24}$. La normalización en la edición de estos textos por parte de E. HüBNER acepta la posibilidad también de una adecuación de todos los personajes a la filiación $L(u c i)$ $f(i l i u s)$, prueba de ello es la integración última que hace de CIL II 2479 (=5617), donde prefiere también $L$ (uci) f(ilius) e insiste de nuevo en la posibilidad de que se trate del historiador citado por Plinio y Solino y en que los Caesares Augusto y Tiberio serían aquellos de los cuales Bocchus fue praefectus ${ }^{25}$. Respecto a la prefectura de los emperadores son importantes G. Mennella ${ }^{26}$ y S. Demougin que opina que $L$. Cornelius Bocchus fue prefecto de Nerón y Druso Césares, los príncipes caídos en desgracia en 31 d.C. por parte de Tiberio ${ }^{27}$.

DeviJVer, como ya hemos señalado, discrepó de la posición que consideró implícita en el corpus de J. D'ENCARNAÇão ${ }^{28}$. La posición unitaria entre ambos Bocchi, sin embargo, fue seguida por L. A. CuRCHIN que, aceptando la filiación C(ai) f(ilius), mantiene como muy probable la identificación del personaje con el historiador romano citado en las obras de Plinio y De Solino, que permitirían, en su opinión, una datación en el reinado de Claudio ${ }^{29}$. A. Stein asume también que se trate de un mismo personaje y que el $L$. $f$. de CIL II 5184, sea un error, y no ve problema en que se esté ante la fuente de Plinio para temas hispánicos y sobre todo de Lusitania y, como ya notó Th. Mommsen, considera menos probable que sea la fuente utilizada por Solino ${ }^{30}$.

Un análisis prosopográfico más reciente abre de nuevo la posibili-

${ }^{24}$ HÜBNER 1872: 182-183.

${ }^{25}$ HÜBNER 1899: 356.

${ }^{26}$ Mennella 1989: 379 y 388, donde señala que se trata del único eques conocido en Hispania que ejerció el cargo de praefectus Caesarum. Cf. además Didu,19831984:63-64, donde parece pensar en un solo personaje y que se tratase de un cargo de Scallabis, recordada como Praesidium Iulium por Plinio (nat. 4, 22,117); los Césares serían en su opinión Gayo y Lucio.

${ }^{27}$ Demougin 1992: 424-425.

${ }^{28}$ DeviJver 1993:2080, dice literalmente: "nobis duo tribuni diversi videntur" refiriéndose a CIL II 5184 y al hecho de que J. d'Encarnacão en IRCP abriera la posibilidad, fortasse, de una identidad entre ambos.

${ }^{29}$ CURCHIN 1990: 177, núm 389, que considera también relacionable con el mismo personaje, como había ya propuesto $I R C P$, su núm. 390 (=IRCP, 188); significativamente CURCHIN precisa: "Some confusión has arisen from the filiation "L. f." in CIL II 5184", lo cual justificaría su opción por leer C. $f$.

${ }^{30} \mathrm{PIR}^{2}$ núm. C 1333, p. 312, (A. Stein).

Conimbriga, 55 (2016) 251-267 
dad de ver tres generaciones de Cornelii Bocchi ${ }^{31}$ : un primer personaje sería Gaius Cornelius Bocchus, CIL II $2479=5617=$ EE VIII $4=$ IRCP 189, documentado en Aquae Flaviae, praefectus Caesarum por dos veces y praefectus fabrum cinco veces, que viviría en el reinado de Tiberio y que es mencionado como flamen perpetuus de la colonia de Scallabis. El segundo sería un Lucius Cornelius Gaifilius Bocchus, CIL II $35=I L S 2920=I R C P 185$, atestiguado en Scallabis, que fue tribuno militar en el reinado de Claudio. El tercero sería Lucius Cornelius Luci filius Bocchus, que habría sido tribuno militar de la legio III Augusta en el reinado de Nerón o en el de Vespasiano, al cual documenta una inscripción, CIL II $5184=I L S 2921=I R C P$ 207, de Caetobriga, Setúbal ${ }^{32}$.

S. Demougin había propuesto, como ya hemos recogido, aceptar la identidad de los dos primeros Cornelii Bocchi citados con el también documentado en Salacia, IRCP $188^{33}$, y aceptaba la distinción de R. ÉtIenNe, siguiendo a J. LeITE DE VASCONCELLOS, entre los dos $L$. Cornelii Bocchi en función de su distinta filiación. Lo que implicaría un largo cursus municipal antes de llegar al ordo equester como ha notado F. Des Boscs-Plateaux, la cual ve dificultades en la atribución al mismo personaje de $\operatorname{IRCP} 188$, que podría convenir también al más reciente de los Cornelii Bocchi en función de la falta de filiación y de la mención del IIviratus. Esta estudiosa termina su argumentación diciendo que uno de los dos personajes, si se acepta la reducción a dos, sería el Bocchus citado por Plinio y recuerda que también Solino se refiere como fuente a un Bocchus en su obra publicada en el año 49 d.C. ${ }^{34}$

Sir Ronald Syme no dejó de hacer también su contribución al expediente de los Bocchi lusitanos al tratar de las fuentes de Plinio y cree evidente que la fuente pliniana fue $L$. Cornelius C.f. Bocchus y mantiene, a pesar de la lectura de R. ÉTIENNE y J. LEITE DE VASCONCELLOS, que el personaje honrado en Salacia por la colonia Scallabitana ( CIL II 35 $=I L S 29200=I R C P 185)$ y el presente en la inscripción de Caetobriga

${ }^{31}$ Des Boscs-Plateaux 2005:629-630, núm. 160.

${ }^{32}$ Des Boscs-Plateaux 2005: 630 y 643, núm. 178.

${ }^{33}$ Demougin, 1992: 423-426, núms. 512-513, donde considera possible que uno de los dos Bocchi pueda ser el citado por Plinio y por Solino. Además Demougin 1988: 837, núms. 512-513.

${ }^{34}$ Des Boscs-Plateaux 2005: 630-631, donde hay un cambio en la numeración de los testimonios que puede inducir a error.

Conimbriga, 55 (2016) 251-267 
$\left(C I L\right.$ II $514=I L S 2921=I R C P$ 207) son el mismo individuo ${ }^{35}$. H. G. Pflaum con su habitual perspicacia se limita a hacer constataciones prosopográficas sobre la dificultad de la secuencia de cargos, pero cree que sea un solo personaje el que citan la inscripción de Salacia y la de Caetobriga a las cuales nos acabamos de referir ${ }^{36}$

Una reciente contribución de J. D'ENCARNAÇão propone identificar con altísima probabilidad cuatro distintos Cornelii Bocchi documen$\operatorname{tados}^{37}$. Con singular agudeza J. DE AlARCÃo propone tres stemmata distintos para establecer las relaciones familiares de los Cornelii Bocchi y señala la dificultad de deducir quién pudo ser el escritor entre los mismos $^{38}$. Los estudios sucesivos de J.C. SaQuete y de M. González HERRERO sobre una nueva inscripción emeritense, de dificil integración, y bien publicada por A. U. STYLOW y A. Ventura y comentada por D. FISHWICK ha ampliado considerablemente el horizonte de la cuestión ${ }^{39}$.

Los Cornelii Bocchi en su condición de caballeros lusitanos han sido objeto también de contribuciones recientes específicas más allá de su presencia en los repertorios generales ${ }^{40}$. La comprensión de su importancia económica en la Lusitania ha avanzado también enormemente a la luz de los hallazgos arqueológicos ${ }^{41}$.

Las hipótesis sobre este tema se encabalgan, como podemos ver, y cobran fuerza a partir de datos conjeturales tomados como premisas. La cuestión de los Bocchi citados por Plinio y por Solino es de por sí espinosa y puede no responder a la existencia no sólo de un único autor sino a la de dos ${ }^{42}$. El hecho de considerar que uno de ellos, el citado por

${ }^{35}$ Syme 1979: 759-760, esp. p. 760 nota 3.

${ }^{36}$ Pflaum 1965: 93.

${ }^{37}$ ENCARNAÇÃO 2011: 196

${ }^{38}$ ALARCÃo 2011: 329-336 y esp. 335.

${ }^{39}$ Stylow y Ventura 2009: 486-489, núm. 11 y p. 521; Saquete 2011: 163172; González Herrero 2013: 403-415. Para el flaminato provincial de los Bocchi Delgado Delgado 1999: 447-449, con una buena bibliografía, y Delgado Delgado 2011: 240. ADEMÁs FisHWicK 2011: 252-254.

40 González Herrero 2002:33-57; GonzÁlez Herrero 2004: 368-370; GonzÁlez Herrero 2006: 33-45; GonZÁlez Herrero 2011:245-258; Caballos RuFino 1998: 214-216, núm.5, y CABallos RuFino 1999: 491, núm.L 5, identificándolo también con la fuente de Plinio el Viejo y Solino.

${ }^{41}$ Morais 2007: 133-136; Morais y Bernardes 2011: 123-131.

${ }^{42}$ Un estado de la cuestión sobre la presencia de Cornelius Bocchus en Plinio el 
Plinio, o ambos puedan identificarse con uno de los Cornelii Bocchi lusitanos es una propuesta interesante, pero hay que considerar que este hecho no tendría otra posibilidad de prueba más allá de la condición de hispana propuesta para la fuente, o fuentes, a partir de los textos especialmente de Plinio y de Solino, que fueron atribuidos a la autoría de un Bocchus lusitano por primera vez por E. HüBNER, que fue seguido por TH. Mommsen ${ }^{43}$. A partir de esta hipótesis de origen hispano de la fuente o fuentes, pesó en ambos estudiosos evidentemente la homonimia de la posible fuente, citada por el cognomen en Solino y por nomen y cognomen en el caso de Plinio el Viejo, con los miembros, dos o tres, conocidos de la familia de Salacia, sin que se pudiera suponer cuál de ellos en ausencia de otros datos. Nos movemos por consiguiente en un terreno conjetural, donde se encabalgan hipótesis plausibles en sí mismas pero que no pueden tomarse como certezas. El nomen Cornelius es de una gran frecuencia y el cognomen Bocchus es en verdad relativamente poco común y con una dispersión geográfica limitada a la Lusitania en las provincias occidentales europeas del imperio romano, aunque no cabe duda de que en la Lusitania, donde parece tener una mayor incidencia, está vinculada principalmente a una sola familia, pero tenemos documentado también Boccus ${ }^{44}$. Por esta razón la dispersión en Lusitania de los Cornelii Bocchi ha sido también objeto de un reciente trabajo ${ }^{45}$. A pesar de ello, en términos comparativos, el resultado no permite situar en aquella zona de forma exclusiva, y mucho menos segura, a los Bocchi, basta solo recordar a los sucesivos reyes de Mauritania de este nombre por no dar más que un ejemplo sobradamente conocido, sobre el que no vale la pena insistir ${ }^{46}$.

\footnotetext{
Viejo en Guerra 2011: 293-305; para la presencia de un Bocchus como fuente de Solino, FERNÁNDEZ Nieto 2011: 307-318, y también Alvar EzQuerRa 2011: 259-274, para la figura literaria, que aceptan todos ellos la identidad del Bocchus escritor con uno de los Bocchi lusitanos.

${ }^{43}$ Así es aceptado por la mayoría de los estudiosos sobre el tema cf. DA Silva Fernandes 2002: 155-171; Almagro Gorbea 2010: 407ss; Almagro 2011: 25-56. Recordemos a título de curiosidad el famoso arículo de Leite de Vascellos 1923-1925, 25, donde recuerda la figura de Cornelius Bocchus y se remite a su publicación de 1895. Véase además la nota anterior.

${ }^{44}$ CIL II 769, de Coria, cf. Atlas:, 116; y CIL II 410, de Viseu, como ejemplo cercano.

${ }^{45}$ D'EnCARnAÇÃo 2011:189-201, con un excelente estado de la cuestión.

${ }^{46}$ Para la dispersión europea occidental de los Bocchi cf. LőRINZ, REDő 1994:05.
}

Conimbriga, 55 (2016) 251-267 
En suma, han pasado más de 30 años y debemos mantenernos todavía, a pesar de los muchos esfuerzos y conjeturas bien fundamentadas pero no probadas, en la prudente conclusión de José D'ENCARNAÇÃO: "Enquanto outros dados não surgirem, essa questão deberá mantener-se em aberto (refiriéndose al Bocchus citado por Plinio), não sendo também fácil aseverar qual o parentesco existente entre este (IRPC 185) e os Bocchi dos n ${ }^{\text {os }} 189$ e $207^{\prime \prime 4}$.

No cabe duda que sobre todo la identificación entre sí de los Boc$c h i$, fuentes respectivas de Plinio y Solino, debe de restar abierta, incluso coincidiendo en valorar el conocimiento hispánico de ambas fuentes y la probable naturaleza lusitana de al menos el autor de una de ellas. Más movedizo es todavía el terreno de la identificación de ambos autores o de uno de ellos con uno de los Bocchi lusitanos, que es una posibilidad que no puede descartarse de plano, pero que dista mucho de tener elementos de prueba fehacientes. Recordaremos por último que Plinio en su Historia natural, 37, 7, 97, dice: Bocchus et in Olisiponensi erui scripsit, magno labore ob argillam soli adusti, refiriéndose a la explotación de los carbunculi; o bien en 37, 2, 24: Cornelius Bocchus et in Lusitania perquam mirandi ponderis in Ammaeensibus iugis, depressis ad libramentum aquae puteis, tratando en este caso del crystallum. Ambas referencias han conducido, sin duda incluso razonablemente, a la situación actual, pero no establecen por una parte la ligazón necesaria con el Bocchus citado en Solino, ni tampoco con los Cornelii Bocchi lusitanos que conocemos, por mucho que podamos pensar que la fuente de Plinio debió de ser muy cercana a los mismos en función de su conocimiento de la Lusitania e incluso de la zona donde éstos parecen haber desenvuelto su actividad. En el estado actual de las cosas sólo una nueva profundización prosopográfica de carácter más general permitirá quizás obtener nuevas precisiones ${ }^{48}$, pero deberemos convenir que por el momento falta todavía el eslabón de unión que pueda asegurar más sólidamente la cadena.

Cf. ahora López CAStro 2011: 113-122, para el posible origen fenicio del cognomen Bocchus.

${ }^{47}$ IRCP: 258.

${ }^{48}$ Muy probablemente, mutatis mutandis, en la línea iniciada por SyME 1979: 742-773.

Conimbriga, 55 (2016) 251-267 
No podemos dudar en afirmar, para concluir, que si no alcanzamos siquiera, en el estado de nuestros conocimientos, a identificar entre ellos a los dos Bocchi citados por las fuentes literarias, mucho menos todavía resulta posible intentar relacionar al menos uno de ellos con uno de los Bocchi documentados epigráficamente en Lusitania, que presentan además a su vez problemas por ahora irresueltos, tanto de individuación como de relación entre ellos a la vista de los documentos inscritos. No cabe duda de la validez de los estudios recientes sobre el tema que han aportado nuevos puntos de vista en apoyo de la intuición de HÜBNER recogida por MomMSEN. Nuestra aportación no pretende ser más que una llamada de atención crítica ante el peso, que creemos excesivo, del criterio de autoridad en esta doble cuestión, literaria y epigráfica, donde hay un confín muy sutil entre el verum y el aptum.

\section{BIBLIOGRAFÍA}

Alarcão, J. de (2011), “Os Cornelii Bocchi, Tróia e Salacia”, en Cardoso y Almagro 2011: 323-347.

Almagro Gorbea, M. (2010), "Lucio Cornelio Boco, Turdetano de Salacia y autor de la Edad de Plata de la Literatura Latina”, Estudos Arqueológicos de Oeiras, 18: 287-332.

Almagro Gorbea, M. (2011), “L. Cornelius Bocchus, político y literato recuperado del olvido", en Cardoso y Almagro 2011: 25-56.

Alvar Ezquerra, A. (2011), "Más notas de asedio a Lucio Cornelio Boccho" en CarDOSO Y ALMAGRo 2011: 259-274.

ATLAS (2003)= Grupo Mérida, Atlas antroponímico de la Lusitania romana, Mérida, Burdeos.

Caballos Rufino, A. (1998), "Los equites y la dinámica municipal de la Lusitania. I. Catálogo prosopográfico”, en L. Hernández Guerra y L. Sagredo San Eustaquio eds., El proceso de municipalización en la Hispania romana. Contribuciones para su estudio, Valladolid: 205-233.

Caballos Rufino, A. (1999), "Los caballeros romanos originarios de las provincias de Hispania. Un avance", en L'ordre équestre. Histoire d'une aristocratie (II siècle a. J.-C. - III e siècle ap. J.-C.). Actes du Colloque international organisé par Ségolène Demougin, Hubert Devijver (†) et Marie-Thérèse Raepsaet Charlier (CEFR, 257), Roma: 464-512.

Cardoso, J.L. y Almagro-Gorbea, M. eds. (2011), Lucius Cornelius Bocchus escritor lusitano da Idade da prata da Literatura Latina. Actas do Coloquio Internacional celebrado em Troia (Outubro de 2010), (Bibliotheca Archaeologica Hispana, 36), Lisboa-Madrid.

Conimbriga, 55 (2016) 251-267 
Curchin, L.A. ( 1990), The Local Magistrates of Roman Spain (Phoenix, Suppl. Vol. XXVIII) Toronto, Buffalo, London.

Delgado Delgado, J.A. (1999), "Flamines provinciae Lusitaniae”, Gerión, 17: 433461.

Delgado Delgado, J.A. (2011), "El flaminado local y provincial en Lusitania. Contribución a la historia política, social y religiosa de una provincia hispana", en Cardoso y Almagro 2011: 231-244.

Demougin, S. (1988), L'ordre équestre sous les julio-claudiens (CEFR 108), Roma.

Demougin, S (1992), Prosopographie des chevaliers romains julio-claudiens (CEFR, 153), Roma.

Des Boscs-Plateaux, F. (2005), Un parti hispanique à Rome? Ascension des élites hispaniques et pouvoir d'Auguste à Hadrien (27 av. J.-C. - 138 ap. J.C.), (Bibliothèque de la Casa de Velázquez, 32), Madrid.

Devijver, H. (1976), Prosopographia militiarum equestrium quae fuerunt ab Augusto ad Gallienum. Pars prima. Litterae A - I (Symbolae Facultatis Litterarum et Philosophiae Lovaniensis, series A, vol. 3), Leuven.

DeviJver, H. (1993), Prosopographia militiarum equestrium quae fuerunt ab Augusto ad Gallienum. Pars quinta. Supplementum II (Symbolae Facultatis Litterarum et Philosophiae Lovaniensis, series A, vol. 3), Leuven.

DiDu, I. (1983-1984), "I praefecti come sostituti di imperatori, cesari et altri notabili eletti alle più alte magistrature municipali”, Annali della Facoltà di Lettere e Filosofia dell'Università di Cagliari, N.S. 5: 53-91.

EnCarnação, J. d' (2011), "Cornelii Bocchi de Olisipo, Scallabis e Salacia”, en CarDOSO y Almagro 2011: 189-201.

Espanca, T. (1966), Inventario artístico de Portugal, vol. VII, Concelho de Évora, Lisboa.

ÉtIENne, R. (1974), Le culte impérial dans la Péninsule Ibérique d'Auguste à Diocletien, Paris (segunda edición,la primera es de 1958), (BEFAR, 191), Paris.

Faria, J.C. Lázaro (1984), Ficheiro Epigráfico, 9: 14-15.

Fernandes, L. da Silva (2002), “Cornelius Bocchus, auctor Lusitanus e notável de Salacia”, en A.A. do Nascimento ed., De Augusto a Adriano. Actas do Colóquio de Literatura Latina (Lisboa 2002), Lisboa: 155-171.

Fernández Nieto, F.J. (2011), "Boco y Solino. L. Cornelius Bocchus en la Collectanea de Iulius Solinus", en Cardoso y Almagro 2011: 307-322.

Ferreira, F. Bandeira (1956), “A inscrição lusitano-romana da Quinta da Sempre- Noiva (Arraiolos) e o problema dos Cornelii Bocchi”, O Arqueologo Português, n.s. III: $87-105$.

Fishwick, D. (2002), The Imperial Cult in the Latin West, 3,2, Provincial Cult: The Provincial Priesthood, (RGMR, 146), Leiden.

Fischwick, D. (2011), "L. Cornelius L. f. Bocchus and the Office of [curator templi Divi] Augusti”, ZPE, 178: 252-254.

GonzÁlez Herrero, M. (2002), “Contribución al estudio prosopográfico de los equi-

Conimbriga, 55 (2016) 251-267 
tes lusitanorromanos: el cursus honorum protagonizado por el tribuno Lucius Cornelius Luci filius Galeria Bocchus", Aquila legionis, 2:33-57.

GonzÁlez Herrero, M. (2004), "Prosopografía de los praefecti fabrum originarios de Lusitania", RPortA, 7: 368-370;

GonzÁlez Herrero, M. (2006), Los caballeros procedentes de la Lusitania romana. Estudio prosopográfico, Madrid.

González Herrero, M. (2011), "La figura de L. Cornelius L. f. Gal. Bocchus entre los praefecti fabrum originarios de Lusitania", en CARdoso y Almagro 2011: 245-258.

González Herrero, M. (2013), "L. Cornelius L.f. Gal. Bocchus y L. Fulcinius Trio: nuevas reflexiones", REA, 115: 403-415.

Guerra, A. (2011), Cornélio Boco. Literato lusitano e Plinio-o-Velho", en Cardoso y Almagro 2011: 293-306.

HüBNER, E. (1866), "Cornelius Bocchus”, Hermes, 1: 397.

HÜBNER, E. (1872), “Additamenta ad Corporis volumen II”, Ephemeris Epigraphica, I, Berlin:182-186.

HüBNER, E. (1899)“Additamenta nova ad Corporis volumen II”, Ephemeris Epigraphica, VIII, Berlin: 351-620.

$I L S=$ H. Dessau, Inscriptiones Latinae Selectae, vol. I, Berlin 1954 ( editio secunda).

$I R C P=\mathrm{J}$. d'Encarnação, Inscrições romanas do conventus Pacensis. Subsídios para o estudo da romanização, Coimbra 1984.

Le Bohec, Y. (1989), La troisième Légion Auguste (Études d'Antiquités Africaines), Paris.

López Castro, J.L. (2011), "Bocchus y la antroponimia fenicio-púnica”, en CARDoso y Almagro 2011: 113-122.

LŐRINZ, B. REDÖ, F. (1994), Onomasticon provinciarum Europae Latinarum. Vol. I: Aba-Bysanus, Budapest (Archaeolingua, 3).

Mennella, G. (1989),"I prefetti municipali degli imperatori e dei cesari nella Spagna Romana”, en C. Castillo ed. (con la col. de J.M. Bañales, R. Martínez y R. Serrano), Actas del Coloquio Internacional A.I.E.G.L. sobre Novedades de Epigrafia Jurídica Romana en el último decenio, Pamplona: 377-389.

Morais, R. (2007), "Contributo para o estudo da economia na Lusitânia romana", Saguntum, 39: 133-140.

Morais, R. Bernardes, J.P. (2011), “L. Cornelius L. f. Bocchus e a economia da Lusitânia”, en Cardoso y Almagro 2011: 123-131.

Peter, H (1906), Historicorum Romanorum reliquiae, vol. II, Leipzig (nos hemos servido de la reimpresión de Stuttgart 1967).

Pflaum, H.-G. (1965), "La part prise par les chevaliers romains originaires d'Espagne à l'administration imperiale", en Les empereurs romains d'Espagne. Madrid - Italica 31 mars - 6 avril 1964, Paris (Colloques internationaux du Centre National de la Recherche Scientifique. Sciences humaines).

Saquete, J.C. (2011), "L. Fulcinius Trio, L. Cornelius Bocchus y el templo del divus Augustus en Mérida”, Habis, 42: 163-172.

Conimbriga, 55 (2016) 251-267 
SchanZ, M. (1901), Geschichte der römischen Literatur bis zum Gestzgebungswerk des Kaisers Justinian (Handbuch der Klassischen Altertumswissenschaf, VIII, 2), München.

Schanz, M. Hosius, C. Krueger, G. (1935), Geschichte der römischen Literatur bis zum Gestzgebungswerk des Kaisers Justinian (Handbuch der Klassischen Altertumswissenschaf, VIII, 2), München.

Stylow, A.U., Ventura Villanueva, Á. (2010), "Los hallazgos epigráficos”, en R. Ayerbe Vélez, T. Barrientos Vera, F. Palma Garcias eds., El foro de Augusta Emerita. Génesis y evolución de sus recintos monumentales (Anejos de Archivo Español de Arqueología, LIII), Madrid: 453-523

Syme, R. (1979), "Pliny the Procurator", en R. Syme, Roman Papers II ( ed. por E. Badian), Oxford: 742-773 (= Harvard Studies in Classical Philology, LXXIII, 1969: 201-236).

Leite de Vasconcellos, J. (1895), "Excursão arqueológica a Alcacer-do-Sal”, $O$ Arqueólogo Português, I: 65-92.

Leite de VASCONCELlos, J. (1914), "Excursão arqueológica à Estremadura Transtagana”, O Arqueólogo Português, XIX: 300-323.

Leite de Vasconcellos, J. (1923-1925), "História da língua portuguesa. Origem e vida externa”, Revista Lusitana, XXV, pp. 5-28.

Conimbriga, 55 (2016) 251-267 